\title{
Diseño, implementación y análisis económico de un Micro Sistema Fotovoltaico Conectado a Red (MSFCR) de 3,2 kWp con inversor-string para fines de investigación en la sede de Ayabacas- UNAJ
}

Design, implementation and economic analysis of a micro grid-connected $3.2 \mathrm{kWp}$ solar PV system with string inverter for research purposes in campus Ayabacas-UNAJ

\author{
Vianey Daysi Chura Palli(1) \\ vd.chura.pa@unaj.edu.pe - Universidad Nacional de Juliaca \\ https://orcid.org/0000-0003-0439-4273 \\ Pedro Yulian Puma Roque ${ }^{(1)}$ \\ py.puma@unaj.edu.pe-Universidad Nacional de Juliaca \\ https://orcid.org/0000-0001-8330-2108 \\ Vilma Sarmiento Mamani ${ }^{(1)}$ \\ v.sarmiento@unaj.edu.pe - Universidad Nacional de Juliaca \\ https://orcid.org/0000-0002-5520-716X \\ Henry Pizarro Viveros ${ }^{(1)}$ \\ h.pizarrov@unaj.edu.pe - Universidad Nacional de Juliaca \\ https://orcid.org/0000-0002-9303-903X
}

(1) Grupo de Investigación y Desarrollo en Tecnologia Solar - GRIDSOLAR/UNAJ

Recibido el 30/10/20 | Aceptado el 20/11/20

DOI: https://doi.org/10.47190/nric.v3i1.133

\section{Resumen}

La generación de energía eléctrica a través de combustibles fósiles contamina el medio ambiente y contribuyen al incremento del efecto invernadero. El uso de energías renovables está cada vez más difundido y es la energía solar fotovoltaica la que presenta mayor crecimiento. La literatura muestra que los sistemas fotovoltaicos conectados a la red (SFCR) son instalados en diversos proyectos, pero a altitudes no mayores de $2500 \mathrm{msnm}$. Sin embargo, en nuestra región no se tiene un SFCR con inversor-string utilizado con fines de investigación para estudiar el comportamiento de estos sistemas fotovoltaicos en las condiciones geográficas y climatológicos de la ciudad de Juliaca. La presente investigación, diseñó, implementó y analizó económicamente un Micro Sistema Fotovoltaico Conectado a la Red (MSFCR) de 3,2 kWp con inversor string para fines de investigación instalado en el Instituto de Energías Renovables y Eficiencia Energética de la Universidad Nacional de Juliaca en su sede Ayabacas. EI MSFCR monofásico cuenta con 12 módulos fotovoltaicos policristalinos de $270 \mathrm{Wp}$ de marca Talesun, un inversor-string de 3 kW de marca Sunny Boy, sistemas de protección en el lado CC y AC, instalado en una estructura metálica diseñada exclusivamente para el montaje del sistema. La producción de energía esperada es $6142,56 \mathrm{kWh} / a n ̃ o$, considerando todas las pérdidas del sistema con un rendimiento global o Performance Ratio (PR) de 85,55\%. Los resultados de los índices económicos muestran que el proyecto es rentable económicamente, el VAN es S/.19985,29 y la TIR es de $16 \%$.

Palabras claves: Inversor string, micro sistema conectado a red (MSFCR), solar fotovoltaica, energía solar.

Como citar: Chura-Palli, V. D., Puma-Roque, P. Y., Sarmiento-Mamani, V. \& Pizarro-Viveros, H. (2021). Diseño, implementación y análisis económico de un Micro Sistema Fotovoltaico Conectado a Red (MSFCR) de 3,2 kWp con inversor-string para fines de investigación en la sede de AyabacasUNAJ. ÑAWPARISUN - Revista de Investigación Científica, 3(2), 13-18. 


\section{Abstract}

The generation of electrical energy through fossil fuels pollutes the environment and contributes to the increase of the greenhouse effect. The use of renewable energies is increasingly widespread and it is photovoltaic solar energy that shows the greatest growth. Literature shows that grid-connected photovoltaic systems (SFCR) are installed in various projects, but at altitudes no higher than 2500 meters above sea level. However, in our region there is no SFCR with an inverter-string used for research purposes to study the behavior of these photovoltaic systems in the geographic and climatological conditions of the city of Juliaca. In the present paper, design, implementation and economic analysis of a micro grid-connected of $3.2 \mathrm{kWp}$ solar PV system with a string inverter for research purposes installed in the Institute of Renewable Energies and Energy Efficiency of the National University of Juliaca at campus Ayabacas. The single-phase MSFCR has 12 polycrystalline photovoltaic modules of $270 \mathrm{Wp}$ from Talesun, a string-inverter of $3 \mathrm{kWp}$ from Sunny Boy, protection systems on the DC and AC side, installed in a metal structure designed exclusively for installation. The expected energy production is $6142.56 \mathrm{kWh} /$ year, considering all the losses of the system with an overall performance or Performance Ratio (PR) of $85.55 \%$. The results of the economic indexes show that the project is economically feasible, the NPV is S/.19985.29 and the IRR is $16 \%$.

Keywords: String inverter, micro grid-connected system, PV solar system, solar energy.

\section{Introducción}

Hoy en día en muchos aspectos de la vida se depende de la energía eléctrica y su consumo crece cada día más. La generación de energía eléctrica a través de combustibles fósiles contamina el medio ambiente, es por la importancia de controlar y mitigar los daños ocasionados por los gases de efecto invernadero (GEI), una de las formas es utilizar fuentes renovables.

La energía solar es la más abundante que existe. En la región central de los andes peruanos los índices de radiación solar son altos, al igual que la radiación solar ultravioleta (Salas, Rojas, Pereira Filho, \& Karam, 2017) y con mayor intensidad en la región sur de los andes (NASA, 2018).

El potencial de energía solar en la sede Ayabacas de la Universidad Nacional de Juliaca (UNAJ) está en los índices más altos como muestra la Figura 1. Este potencial solar debe ser aprovechado con proyectos que investiguen las mejores condiciones de sistemas fotovoltaicos y sus implementos para recomendar a la sociedad las tecnologías más óptimas. Sin embargo, estos proyectos son todavía escasos y su implementación servirá para investigar las mejores tecnologías de generación fotovoltaica y de inversores bajo condiciones extremas del altiplano sur peruano.

El inversor-string es tradicionalmente utilizado en diversos sistemas fotovoltaicos en el Perú. Se trata de un inversor descentralizado que trabaja con una

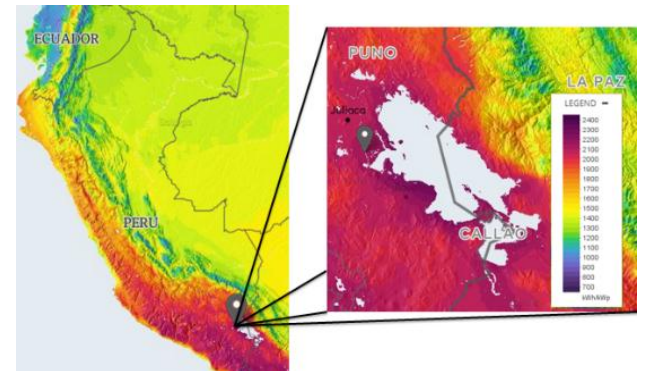

Figura 1. Potencial de energía solar del lugar de instalación (GlobalSolar, 2016). cadena de módulos fotovoltaicos (llamada también string se debe notar la diferencia). El arreglo de módulos fotovoltaicos se conecta a un solo inversorstring por lo cual este tipo de tecnología es más viable económicamente además de tener control óptimo del seguidor del punto de máxima potencia (MPPT) (Teles, 2017). Una desventaja de este arreglo de módulos es el sombreamiento porque cuando un módulo está sombreado afecta toda la cadena de módulos fotovoltaicos y podría reducir el rendimiento del sistema.

Habitualmente los sistemas fotovoltaicos en el Perú utilizan el inversor-string, un ejemplo cercano es el SFCR instalado en el pabellón académico de la UNAJ, que analizó parámetros eléctricos con mediciones experimentales (Torres-Yucra, et al., 2020). Un aspecto importante es el monitoreo de datos en tiempo real en SFCR con inversor-string, una alternativa estudiada es realizado mediante las señales wireless muestran la importancia de monitoreo remoto con altas precisiones (Kabalci \& Kabalci, 2018).

Un sistema fotovoltaico con inversor-string instalado a nivel del mar obtuvo pérdidas totales de $6 \%$ entre pérdidas medidas y proyectadas con el sistema generando a condiciones nominales. La eficiencia obtenida del $97,15 \%$ es ligeramente mayor que las especificaciones técnicas (Fischer \& Uicich, 2018). Las fallas en módulos fotovoltaicos de tres tipos de inversores de los cuales uno es el inversor-string son analizadas para determinar el impacto en la confiabilidad del SFCR concluyendo que el inversorstring tiene el segundo periodo de recuperación que lo convierte en un proyecto medianamente rentable (Tariq, Butt, \& Khan, 2018). Un análisis de eficiencia experimental entre módulos fotovoltaico instalado a 800 msnm con inversor-string y microinversor muestran que la eficiencia en condiciones de sombreamiento y suciedad se reduce respecto a los microinversores (Godoi, 2018).

En el presente artículo, se presenta el diseño e implementación y un análisis económico de un Micro Sistema Fotovoltaico Conectado a la Red (MSFCR) de $3 \mathrm{kWp}$ con inversor-string a condiciones extremas del altiplano sur peruano (radiación solar extrema, frío 
extremo con fuertes vientos) y destinado a aplicaciones de investigación en los laboratorios del Instituto de Energías Renovables y Eficiencia Energética en la sede Ayabacas de la Universidad Nacional de Juliaca (UNAJ).

\section{Metodología}

Para el desarrollo del diseño fueron utilizadas diversas fuentes como la NASA, SENAMHI y mediciones anteriores especializada. La implementación del MSFCR siguió el diseño proyectado. el MSFCR con inversor-string es un sistema monofásico y está conformado por 12 módulos fotovoltaicos de $270 \mathrm{Wp}$ de marca Talesun con una potencia instalada total de $3,2 \mathrm{kWp}$. El inversor-string de $3 \mathrm{kWp}$ de marca Sunny Boy, tableros de protección y un medidor de energía de un solo flujo. Las pérdidas por temperatura fueron calculadas y se estimó la producción de energía anual esperada por el MSFCR.

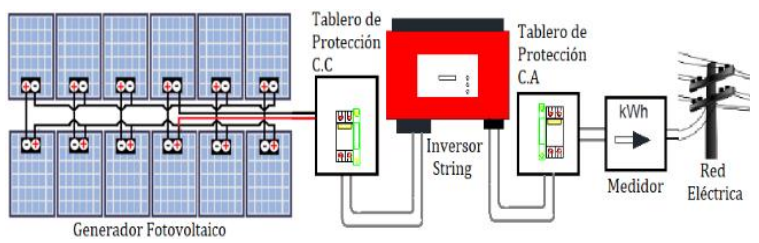

Figura 2. Instalación un Sistema Fotovoltaico Conectado a la Red con inversor-string.

\section{Ubicación geográfica}

El MSFCR de 3,2 kWp está instalado en el Instituto de Energías Renovables y Eficiencia Energética en la sede Ayabacas de la UNAJ, ubicado en el departamento de Puno, en los andes sur peruano $\left(15.22^{\circ} \mathrm{S}, 70.03^{\circ} \mathrm{W}\right.$ ) (NREL, 2020). El lugar de instalación está a una altitud de $3825 \mathrm{~m}$, y está sometido a condiciones ambientales extremas con presencia de radiación solar extrema, frío intenso y vientos fuertes con polvo.

\section{Análisis de sombras}

El sombreamiento en los sistemas fotovoltaicos es un problema constante debido a que disminuyen la potencia de generación. Varias causas son las que ocasionan las sombras en un módulo fotovoltaico, sombras de los árboles, edificios de vecinos, clima nublado, sombras y otros módulos fotovoltaicos adyacentes. El análisis de sombras fue realizado con el software Sketchup (Zomer \& Ruther, 2017a) (Zomer \& Ruther, 2017b) comprobándose que ningún objeto de la edificación interferirá con el sistema.

\section{Topología del MSFCR}

La utilización de las topologías en los sistemas fotovoltaicos deben garantizar que la corriente esté en fase con la tensión y que sean ondas sinusoidales de alta calidad y con baja distorsión (Baekhoej, Pedersen, \& Blaabjerg, 2005). El MSFCR ocupa un área de $15 \mathrm{~m}^{2}$, tiene una configuración de cadena en serie con un solo inversor-string. Posee un sistema de protección en los lados $\mathrm{CC} / \mathrm{AC}$ y un medidor de energía de un solo flujo.

\section{Ángulo de inclinación}

El ángulo de inclinación utilizado es igual a la latitud de la ubicación del lugar de instalación (Jacobson \& Vijaysinh, 2018). Considerando la alta radiación en los andes sur peruano, las instalaciones de los paneles fueron realizadas a un ángulo fijo para todas las estaciones del año y orientado al norte. Se consideró la irradiación anual en el plano inclinado (15으 de inclinación) de 6,0 kWh/m2-dia.

\section{Especificaciones de los módulos fotovoltaicos}

Los módulos fotovoltaicos utilizados son de tipo policristalino de $270 \mathrm{Wp}$. El voltaje de circuito abierto (Voc) de $38.5 \mathrm{~V}$ y corriente de corto circuito de 9,09 A. Temperatura máxima de operación de $85^{\circ} \mathrm{C}$. Los módulos fotovoltaicos están soportados por una estructura metálica con una altura inicial de $659 \mathrm{~mm}$ y altura final de $1403 \mathrm{~mm}$, que permitirá una fácil limpieza de los paneles.

\section{Inversor-string}

El inversor es un elemento importante en los sistemas fotovoltaicos. El inversor convierte la corriente continua procedente del generador fotovoltaico en corriente alterna. La salida del inversor-string se sincroniza automáticamente con el mismo voltaje y frecuencia de la red a la que se conecta. En la salida del inversor-string, la tensión nominal es $220 \mathrm{~V}$ con una corriente máxima de $16 \mathrm{~A}$

\section{Análisis de pérdidas en el MSFCR}

Las pérdidas que presenta el sistema fueron calculadas y analizadas. Pérdidas por dispersión de potencia, pérdidas por temperatura, por suciedad en módulos fotovoltaicos, por degradación fotónica y pérdidas por potencia del inversor.

\section{Análisis económico}

El análisis de viabilidad económica fue evaluada a través de los siguientes indicadores económicos:

Valor Actual Neto (VAN) mostrada en la Eq. (1),

VAN $=-I+\sum_{i=1}^{n} \frac{Q_{n}}{(1+r)^{n}}$

donde, $Q_{n}$ representa los flujos de caja, I es el valor del desembolso inicial de la inversión, $n$ es el número de periodos considerados, $r$ es la tasa de descuento.

La Tasa Interna de Retorno (TIR), mostrada en la Eq. (2),

$\operatorname{VAN}=-1 \sum_{\mathrm{l}=1}^{\mathrm{n}} \frac{\mathrm{Q}_{\mathrm{n}}}{(1+r)^{\mathrm{n}}}=0$ 
donde, $r$ es igual a la TIR.

Plazo de recuperación (Payback) mostrada en la Eq. (3),

$P S R=a+\frac{(b-c)}{d}$

donde, a es el año inmediato anterior en que se recupera la inversión, b es la inversión inicial, c es el flujo de efectivo acumulado del año inmediato anterior en el que se recupera la inversión, d es el flujo de efectivo del año en el que se recupera la inversión.

\section{Relación Beneficio/Costo (B/C) Eq. (4).}

Compara directamente los beneficios y los costos de un proyecto para definir su viabilidad. El Índice B/C se halla sumando todos los beneficios descontados, traídos al presente, y se divide sobre la suma de los costos también descontados.

\section{Resultados}

Fue diseñado el MSFCR de 3,2 kWp para fines de investigación e instalado en la terraza del Instituto de Energías Renovables y Eficiencia Energética en la sede Ayabacas. Fueron consideradas las condiciones geográficas extremas del lugar de instalación como alta irradiancia solar. Se realizó el análisis de sombras utilizando el software Sketchup ubicando el lugar óptimo de instalación (Figura 3), las sombras que afectarán al sistema serán de tipo climatológico por la presencia de nubes. El ángulo de inclinación fue de $15^{\circ}$ dirigido al norte geográfico del lugar de instalación. La topología del MSFCR resultó en 12 módulos fotovoltaicos de $270 \mathrm{Wp}$ instalado en una estructura metálica diseñada exclusivamente para el montaje del sistema (Figura 4). El sistema de protección proyectado para los lados DC y AC considera protección contra descargas atmosférica a través de un dispositivo de protección contra sobretensiones (DPS). El inversor-string de tipo monofásico.

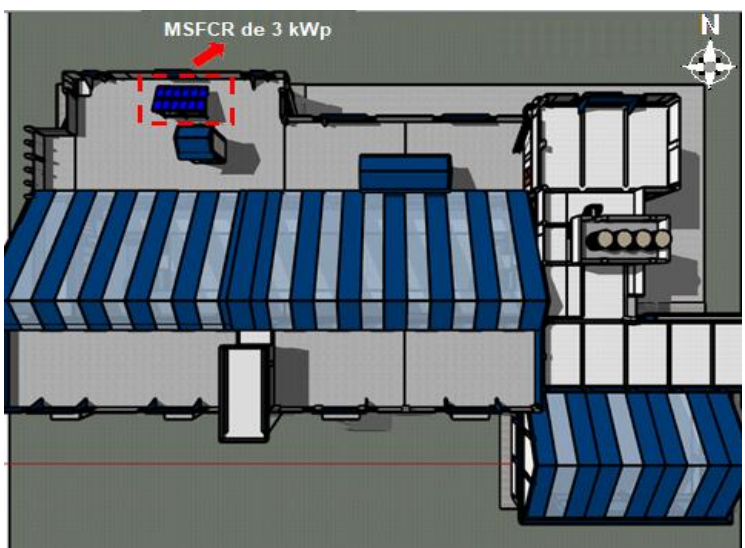

Figura 3. Análisis de sombras mediante Sketchup.

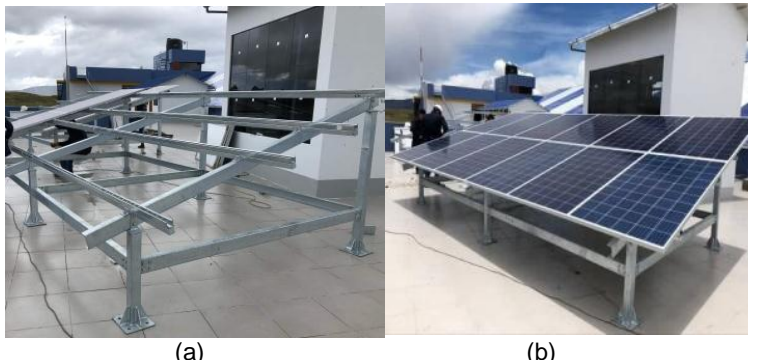

Figura 4. Sistema de generación fotovoltaica: (a) estructura de soporte; (b) montaje de módulos fotovoltaicos.

El diagrama unifilar del MSFCR con el detalle del generador fotovoltaico, el sistema de protección en el lado $\mathrm{CC}$ y lado $\mathrm{AC}$, y el inversor-string son mostrados en la Figura 5.

Las pérdidas del MSFCR calculadas son: mayores pérdidas por temperatura en el mes de octubre con 4,58\%. Pérdidas por dispersión, degradación fotónica y suciedad, $2,5 \%$. Pérdidas por inclinación y por sombreamiento de nubes u otros factores climáticos, haciendo un total de pérdidas de $5,37 \%$. Con una eficiencia del inversor-string de 94,63\%.

Considerando todas las pérdidas del MSFCR el rendimiento global del sistema también llamado Performance Ratio (PR) resultó en 85,55 \%. La máxima producción de energía estimada se da en el mes de noviembre con 596,26 kWh/mes y la mínima se da en el mes de febrero con 440,83 kWh/mes. La producción anual es de 6142,56 kWh/año. La Tabla 1, muestra la producción anual de energía del MSFCR de $3 \mathrm{kWp}$, estando el mes con mayor generación de energía señalado en negrita.

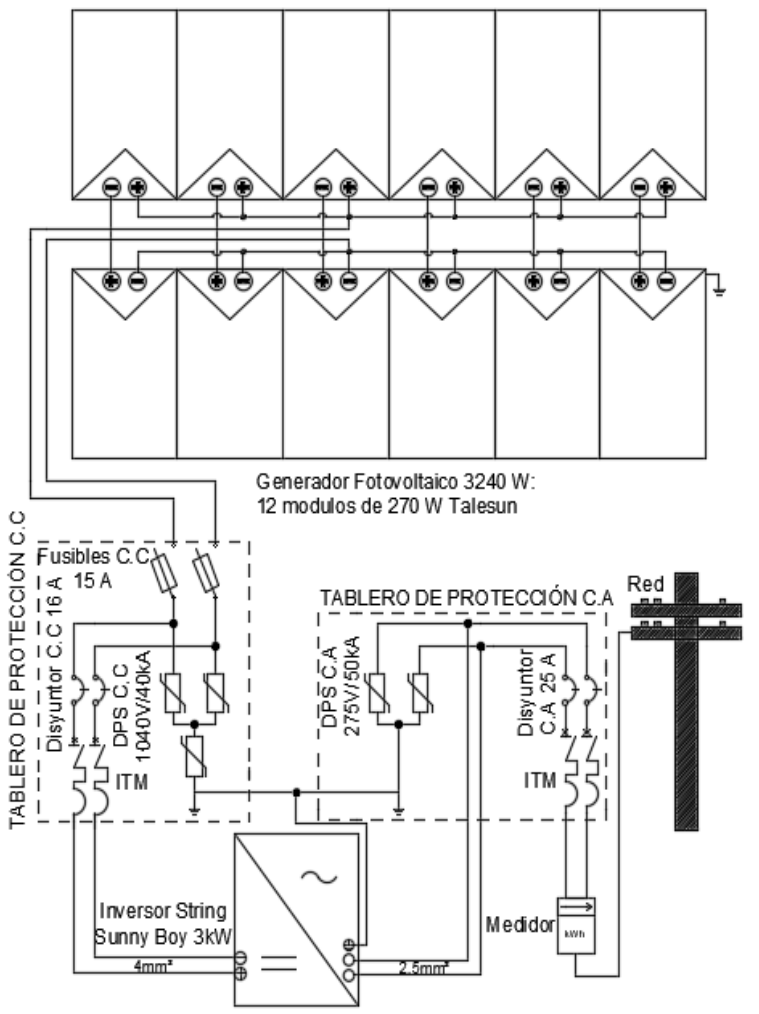

Figura 5. Diagrama unifilar del MSFCR. 
Tabla 1.

Producción anual de energía esperada del generador fotovoltaico de $3 \mathrm{kWp}$

\begin{tabular}{|c|c|c|c|c|c|c|}
\hline Mes & $\begin{array}{l}\mathrm{H}(\alpha, \beta) \\
\left(\mathrm{kwh} / \mathrm{m}^{2 *} \text { día }\right)\end{array}$ & $\begin{array}{l}\frac{H(\alpha, \beta){ }^{*} P_{\mathrm{GFV}}}{\mathrm{G}_{\mathrm{STC}}} \\
\left(\mathrm{kwh} / \mathrm{m}^{2}{ }^{*} \text { día }\right)\end{array}$ & PR & $\frac{E_{P}}{(k w h / \text { día })}$ & Día/mes & $\begin{array}{c}\text { Producción } \\
\text { mensual } \\
\text { (kWh/mes) }\end{array}$ \\
\hline Enero & 5,62 & 18,21 & 0,863 & 15,72 & 31 & 487,32 \\
\hline Febrero & 5,61 & 18,18 & 0,866 & 15,74 & 28 & 440,83 \\
\hline Marzo & 5,45 & 17,66 & 0,858 & 15,43 & 31 & 478,33 \\
\hline Abril & 5,73 & 18,57 & 0,855 & 15,15 & 30 & 454,50 \\
\hline Mayo & 6,11 & 19,79 & 0,851 & 16,84 & 31 & 522,10 \\
\hline Junio & 5,80 & 18,79 & 0,856 & 16,08 & 30 & 482,53 \\
\hline Julio & 5,98 & 19,37 & 0,857 & 16,60 & 31 & 514,60 \\
\hline Agosto & 6,72 & 21,77 & 0,846 & 18,42 & 31 & 570,94 \\
\hline Setiembre & 6,29 & 20,38 & 0,849 & 17,30 & 30 & 519,08 \\
\hline Octubre & 6,96 & 22,55 & 0,845 & 19,05 & 31 & 590,69 \\
\hline Noviembre & 5,95 & 19,28 & 0,858 & 16,54 & 30 & 596,26 \\
\hline Diciembre & 5,64 & 18,27 & 0,857 & 15,66 & 31 & 485,38 \\
\hline Producción & l de energía $(\mathrm{kWh} / \mathrm{arr}$ & & & & & 6142,56 \\
\hline
\end{tabular}

Los resultados del análisis económico muestran la rentabilidad económica del proyecto. La inversión será recuperada en el año 11. El flujo de caja del proyecto fue calculado para un tiempo de 25 años. El VAN es $\mathrm{S} / .19985,29$. El performance ratio (PR) es de $85,55 \%$, con lo cual se espera obtener una producción anual de 6142,56 kWh de energía eléctrica. El TIR es de $16 \%$. El detalle de los índices económicos es mostrado en la Tabla 1.

\section{Conclusiones}

Fue diseñado, implementado y analizado económicamente un MSFCR de 3,2 kWp con inversorstring en el Laboratorio de Energías Renovables y Eficiencia Energética de la sede Ayabacas de la UNAJ. Para el diseño fue considerado la irradiación anual en el plano inclinado (15을 de inclinación) de 6,0 $\mathrm{kWh} / \mathrm{m} 2$-dia. El MSFCR tiene 12 módulos fotovoltaicos policristalinos de $270 \mathrm{~W}$ de marca Talesun.

Tabla 2.

Resultados del cálculo de los Índices económicos.

\begin{tabular}{|c|c|c|}
\hline Indicador Económico & Unidad & Valor \\
\hline Valor Actual Neto (VAN) & $\mathrm{S} /$. & 19985,29 \\
\hline Tasa Interna de Retorno (TIR) & $\%$ & 16 \\
\hline Relación Beneficio Costo & $\mathrm{B} / \mathrm{C}$ & 1,76 \\
\hline $\begin{array}{l}\text { Periodo de Recuperación de la } \\
\text { Inversión Payback con tasa de } \\
\text { descuento }\end{array}$ & años & 10 \\
\hline
\end{tabular}

El inversor-string de $3 \mathrm{kWp}$ de marca Sunny Boy. Tiene sistema de protección en los lados de CC y AC. Fueron calculadas las pérdidas del sistema fotovoltaico obteniéndose un rendimiento global 0 performance ratio (PR) de $85,55 \%$, con lo cual se espera obtener una producción anual de 6142,56 kWh de energía eléctrica.

Según muestran los índices económicos el proyecto es rentable económicamente. El VAN es S/.19985,29 y el TIR es de $16 \%$. El performance ratio (PR) es de $85,55 \%$, para una producción anual de $6142,56 \mathrm{kWh}$ de energía eléctrica. 


\section{Referencias bibliográficas}

Famoso, F., Lanzafame, R., Maenza, S., \& Scandura, P. (2015). Performance comparison between micro-inverter and string-inverter photovoltaic systems. 69th Conference of the Italian Thermal Engineering Association (págs. 526-539). Energy Procedia.

Fischer, F., \& Uicich, S. (2018). Diseño e implementación de un inversor tipo string para inyección a red en sistemas renovables. Mar del Plata. Argentina: Universidad Nacional de Mar del Plata.

GlobalSolar, A. (2016). https://globalsolaratlas.info. (World Bank Group) Recuperado el 23 de Junio de 2020, de https://globalsolaratlas.info/?c=$15.2241067,-70.03456055,7 \& s=-15.749963,-$ 70.004883

Godoi, R. (2018). Análise comparativa de desempenho de inversor String e Microinversor. Uberlândia - Minas Gerais, Brasil.: Universidade Federal de Uberlândia - UFU.

Jacobson, M., \& Vijaysinh, J. (2018). World estimates of PV optimal tilt angles and ratios of sunlight incident upon tilted and tracked PV panels relative to horizontal panels. Solar Energy, págs. 55-56.

Kabalci, E., \& Kabalci, Y. (2018). A wireless metering and monitoring system for solar string inverters. Electrical Power and Energy Systems, págs. 282-295.

NASA. (2018). (National Aeronautics and Space Administration),. (NASA Langley Research Center Atmospheric Science Data Center surface meteorology and solar energy (SSE)) Recuperado el 23 de 05 de 2020, de https://eosweb.larc.nasa.gov

NREL, (. (2020). PV Watts Calculator. Recuperado el 13 de 08 de 2020

Salas, L., Rojas, J., Pereira Filho, A., \& Karam, H. (2017). Ultraviolet solar radiation in the tropical central Andes $\left(12.0^{\circ}\right.$ S). Photochemical \& Photobiological Sciences, págs. 16(6), 954-971.

Tariq, M., Butt, S., \& Khan, H. (2018). Impact of module and inverter failures on the performance of central, string, and micro-inverter PV systems. Microelectronics Reliability, págs. 1042-1046.

Teles, M. (2017). Avaliação operacional das diferentes arquiteturas de interligação de geradores fotovoltaicos à rede elétrica. Belém-Pará, Brasil: Programa de Pós-Graduação em Engenharia Elétrica - Universidad Federal do Pará.

Torres-Yucra, J., Condori-Ramos, J., Condori-Yucra, R., Limachi-Viamonte, W., Pari-Pari, E., \& Beltrán-Castañón, N. (2020). Análisis y evaluación de un sistema fotovoltaico de $3.0 \mathrm{~kW}$ conectados a la red del Pabellón Administrativo de la Universidad Nacional de Juliaca. Ñawparisun - Revista de Investigación Científica, 2(4), 73-80.

Zomer, C., \& Ruther, R. (2017a). Simplified method for shading-loss analysis in BIPV systems - Part 1: Theoretical study. Energy and Buildings, págs. 130.

Zomer, C., \& Ruther, R. (2017b). Simplified method for shading-loss analysis in BIPV systems Part 2: Application in case studies. Energy and Buildings, págs. 1-35.

\section{Agradecimientos}

Este trabajo fue financiado por el CONCYTECFONDECYT en el marco de la convocatoria E041-01 número de contrato $\mathrm{N}^{\circ}$ 180-2018-FONDECYT-BMIADT-AV.

Al Grupo de Estudos e Desenvolvimento de Alternativas Energéticas de la Universidade Federal do Pará (GEDAE/UFPA) Brasil. A los integrantes del grupo de investigación GRIDSOLAR/UNAJ por el esfuerzo y dedicacion al proyecto. 